\title{
Always returning: feedback and sensory processing in visual cortex and thalamus
}

\author{
Adam M. Sillito, Javier Cudeiro, Helen E. Jones
}

Feedback projections are an integral part of the mammalian visual system. Although it is tempting to relegate them to a subsidiary role in visual processing, because their supposed latency and lag might appear to be unfavourable for an involvement in fast processing, this is a dangerous simplification. Certainly for the world in motion, feedback from higher motion areas can influence the transfer of ascending input when, or even before, the input arrives. Here, we consider the circuit formed by layer 6 feedback cells in the visual cortex and how this straddles the retinothalamic and thalamocortical transfer of visual input. We discuss its links to feedback from the cortical motion area MT (V5), and suggest that motion perception involves a dynamic interplay between MT, V1 and the thalamus. This review is part of the TINS special issue on The Neural Substrates of Cognition.

\section{Introduction}

It has long been recognized that the ascending sensory pathways in the brain are paralleled by descending feedback pathways, but there has been little consensus as to the role of the feedback connections in perception. Indeed, most accounts of the visual system still consider the mechanism of vision in terms of a sequential elaboration of processing steps through a hierarchy of visual areas, albeit in parallel interacting systems that at least partially segregate specific channels of information such as motion, form and colour. Alongside such models, a detailed picture of the anatomical organization and functional influence of the feedback pathways is emerging, but a consensus on how this feedback is integrated into roles linked to the feedforward pathways has not yet been reached. This failure is particularly challenging in the early stages of the visual pathway because it is already readily apparent that the synaptic organization of the feedforward and feedback connections forms a circuit, not sets of connections that can be logically segregated according to function. What does this mean? Well, it means for example that the local processing of the visual input in either the visual thalamus (lateral geniculate nucleus, LGN) or layer 4 of the primary visual cortex (V1) is influenced by the spatial context and analysis carried out by higher levels in the system. It also means that there must be seamless feedback-driven modulation of the responses of neurons at earlier levels in the visual system, which in turn changes the input to the neurons proving the feedback, and hence changes their response and thus the feedback. As a generalization, neurons at higher levels in the visual system sample larger areas of visual space (via convergent input from neurons at earlier levels) and the feedback consequently has the ability to phrase local processing of the visual input at earlier levels in the broader spatial context of the higher level. Feedback from the cortical area MT (V5) to layer 6 of V1 is particularly interesting because it has the potential to influence the feedback to the LGN directly. For the world in motion, it could enable predictive modulation of circuitry at earlier levels, even before the input arrives. Such implementation of 'top down' processing might be a fundamental feature of perceptual mechanisms.

\section{Feedback connections}

To elaborate these points further, a useful place to start is the interaction between V1 and the LGN (Figure 1a). Feedforward connections from the LGN target layer 4 and 6 of V1. Cells in layer 6 of V1 provide an extensive feedback connection to the LGN, where terminals from feedback cells comprise $\sim 30 \%$ of the synaptic input to LGN relay cells, compared with $\sim 10 \%$ from retinal afferents 1, 2, 3 and 4. The feedback connections from layer 6 cells in V1 target the distal dendrites of relay cells and also contact inhibitory interneurons in the LGN and the perigeniculate nucleus 5, 6, 7 and 8. The same layer 6 cells also send collateral connections up to layer 4, where the LGN afferents contact the cortex. At this location the layer 6 cell axons again provide a much larger proportion of the excitatory synaptic input (45\%) than the ascending afferents from the LGN (6-9\%) 9 and 10. Thus, the layer 6 cells seem to have a special role as a 'gatekeeper' regulating the transfer of the retinal input to the central visual system. It is 
therefore intriguing that area MT 11, 12 and 13 provides feedback to both the upper and lower parts of layer 6 in V1 14 and 15. This projection can contact all sets of layer 6 cells that provide feedback to the LGN in primate V1, and hence can give the 'motion' signal re-entrant access to all the channels of information relaying through the LGN (the slow-conducting parvocellular system linked with fine form and colour, the slow-conducting konicellular system associated in part with colour, and the fastconducting magnocellular system associated with motion and form from motion) 16 and 17. Given the way that the layer 6 cells straddle the transfer of the retinal input to the visual cortex, the feedback from MT to layer 6 in V1 has to be seen as a fast and potentially potent influence on early visual processing. How can we begin to unravel the way in which the various components of this circuit contribute to function and ultimately perception? A good place to start is to try and find out what the layer 6 cells do.

(a)

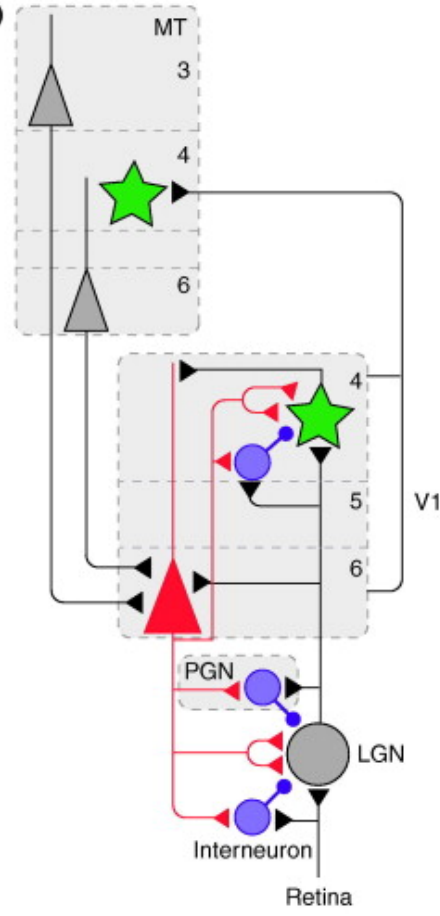

(b)

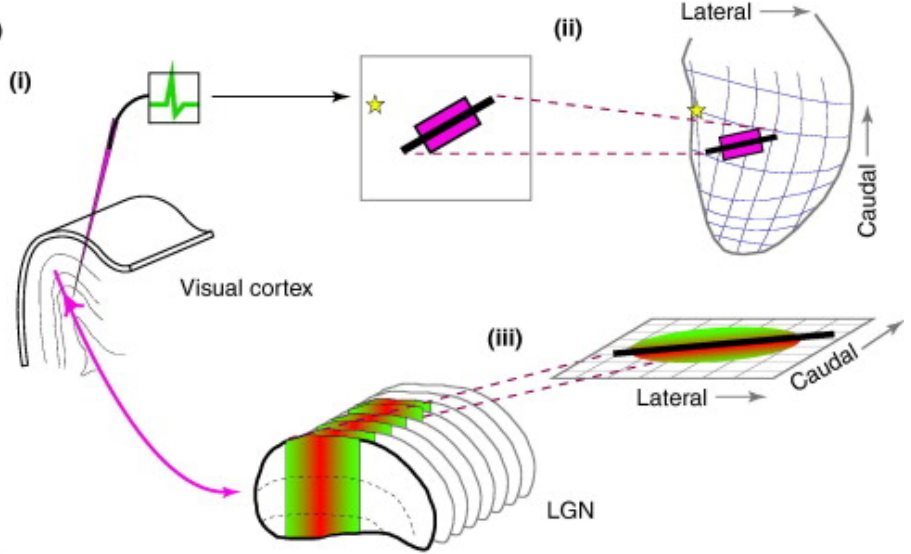

(c)

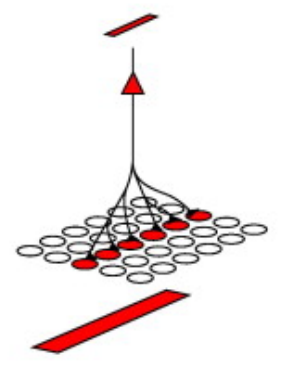

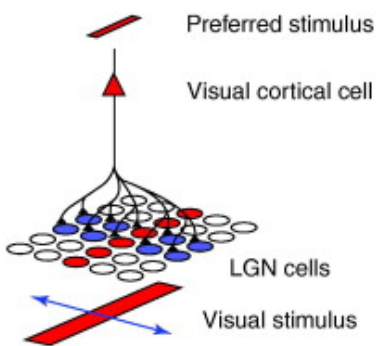

TRENDS in Neurosciences

Figure 1. Anatomical organization of feedback connections. (a) The neural circuit centred on layer 6 cells in the visual cortex influences the transfer both of retinal input to the lateral geniculate nucleus (LGN) and of input from the LGN relay cells to the visual cortex. This summary is to illustrate the concept only and not the detail of the connections. Retinal afferents make synaptic contacts on both relay cells (grey circle) and GABAergic intrinsic inhibitory interneurons (blue) in the LGN. LGN relay cells in turn provide input to both excitatory (green) and inhibitory (blue) neurons in layer 4 of the visual cortex. They also provide collateral input to layer 6 of the visual cortex and to inhibitory neurons in the perigeniculate nucleus (PGN, the visual part of the thalamic reticular nucleus). The PGN cells and intrinsic inhibitory interneurons of the LGN both provide inhibitory input to LGN relay cells. V1 layer 6 cells (red) provide a massive feedback projection to the LGN that contacts relay cells, intrinsic inhibitory interneurons and PGN cells. The same layer 6 cells also send an extensive collateral projection to layer 4 of the visual cortex, where they contact the spiny stellate cells receiving the input from the LGN and local inhibitory interneurons. The layer 6 cells thus appear to exert a unique control over the access of the retinal input to the central visual system. There is also direct feedback from the motion area MT/V5 to layer 6. Cells in layer 4B and 6 of V1 provide a direct feedforward projection to MT completing a circuit of interaction (this diagram only summarizes the direction of the connections; feedback from MT also targets layer 4B). (b,c) Retinotopic asymmetries in the distribution of feedback terminals link to physiological response properties of the parent cells. (b) Summary of method. (i) Cells in layer 6 of the visual cortex were recorded, mapped and labelled with biocytin. The receptive field of the layer 6 cell is denoted by the red rectangle and its preferred orientation is indicated by the thick bar. The star represents the position of area centralis. (ii) The receptive field of the layer 6 cell is shown overlaid on the geniculate retinotopic map. The star represents location of area centralis. (iii) The bouton distribution of single axons was reconstructed in 3D and analysed with respect to the geniculate representation of visual space. (c) Summary of data. Feedback axons contacted LGN cells lying in a line either parallel to (left) or perpendicular to (right) the axis of the orientation preference of the parent layer 6 cell. This suggests a functional focus on either the orientation of the parent cell or the direction of motion of an object crossing the receptive field. See main text for further discussion. Panel (b) modified from Ref. [19]. 
Functional aspects of the role of layer 6 feedback cells

How layer 6 cells respond to visual stimuli and the potential specificity of their effects are key issues. Layer 6 cells exhibit low spontaneous activity, mainly have 'simple'-type receptive fields and are sensitive to stimulus orientation and direction of motion [18]. Thus, the moment by moment properties of the feedback to the LGN will depend on how the visual input drives the layer 6 cell receptive fields. Also, because the feedback projection is retinotopically organized 6, 7 and 19, the spatial focus of the feedback influence, and the stimulus feature-dependent characteristics, will be determined by the details of the stimulus falling on the retina. Thus the feedback, far from being unspecific, will follow the temporal and spatial characteristics of the visual input translated through the stimulus-selective receptive fields of the visual cortex.

Given this dependence on the visual stimulus, the effects of the feedback might be expected to influence LGN relay-cell visual responses in a manner that relates to those stimulus features. So from this position, it is apparent that we could gain insight into the influence of feedback by comparing the visual response properties of LGN cells to these stimulus features in the presence and absence of feedback. Another approach is focal enhancement of layer 6 cell visual responses by local drug application. This has the potential to reveal the influence of feedback by showing what happens when the feedback from one location in V1 gets stronger.

Whatever strategy is used to reveal its influence, it is clear that feedback from V1 does affect the visual response properties of LGN cells 20, 21, 22, 23, 24, 25, 26 and 27. LGN cells, like retinal ganglion cells, are generally not selective for orientation or direction, and they have circular concentric centresurround receptive fields in which responses from stimulation of the centre are antagonized by simultaneous stimulation of the surround. In particular, feedback seems to modulate the strength of this centre-surround interaction for moving stimuli. For example, a simple stimulus such as a short moving bar that is roughly the same size as the centre of an LGN-cell receptive field will provoke a strong response as it crosses the centre of the receptive field. However, if the bar is made longer so that it also covers the surround, the response of the LGN cell will be reduced because of the surround antagonism [28]. Feedback seems to make this surround antagonism for moving stimuli stronger and to reduce the response further [23]. This is manifest by data showing that with feedback, LGN cells in the cat exhibit responses that are biased to short as opposed to long moving bars 23 and 28 and to small as opposed to large patches of drifting grating 25 and 29 (Figure 2 and Figure 3). This property is also referred to as end-stopping, and we discuss it later in relation to MT and V1 receptive-field properties and the perception of the direction of motion of objects as a whole.

This feedback-driven enhanced surround antagonism in LGN cells also includes an increase in the sensitivity to differences in the spatial parameters of stimuli overlying the receptive-field centre and surround 24, 25, 26 and 27. With feedback, LGN cells show a larger difference in firing when the orientation or temporal frequency of a visual stimulus, such as a sinusoidal grating overlying the receptive-field surround, deviates from that of a patch of grating overlying the receptive-field centre 24, 25 and 26 (Figure 3a-c). A recent theoretical model [30] successfully captures this sensitivity to orientation discontinuity and its dependence on feedback from the visual cortex. It is important to reiterate that these effects were seen only with moving stimuli and not with stationary flashing stimuli [23]. One could argue this to be a fine-scale pop-out phenomenon (where a contrasting context causes a stimulus to stand out from the background) linked only to moving stimuli. Although this has not yet been observed in the primate (macaque) LGN, and some doubt its presence in the marmoset LGN [31], the same type of effect has been seen in non-oriented primate (macaque) layer 4C $\beta$ cells in V1 [32] (e.g. Figure 3d). It could be that this processing step is deferred from the LGN in the macaque to the nonoriented cells in layer 4C (which are not seen in cat V1) but it still follows from the influence of layer 6 cells (via their collaterals in layer 4C). It is worth noting that primate V1 cells in general show strong surround suppression 33 and 34, and even oriented simple cells when tested using a very small stimulus over their receptive-field centre show similar orientation-contrast responses to those of non-oriented 4C $\beta$ cells and cat LGN cells 32 and 33. The presence of this effect and its modulation via feedback in macaque LGN have not yet been explored but there are, as we will discuss, clear feedback effects in terms of modulating response magnitude in the macaque LGN, and this pattern of effect might be seen at cortical and thalamic levels. In relation to the potential influence of MT on this system, the point we would make here is that feedback might influence the level of end-stopping and/or pop-out seen in LGN cells (and potentially primate layer 4C cells) to moving stimuli. It has been suggested that end-stopped receptive fields provide a mechanism for resolving the aperture problem in pattern motion perception and that the receptive fields of MT cells integrate the inputs from earlier-level end-stopped cells 13 and 35. We will return to this later. 

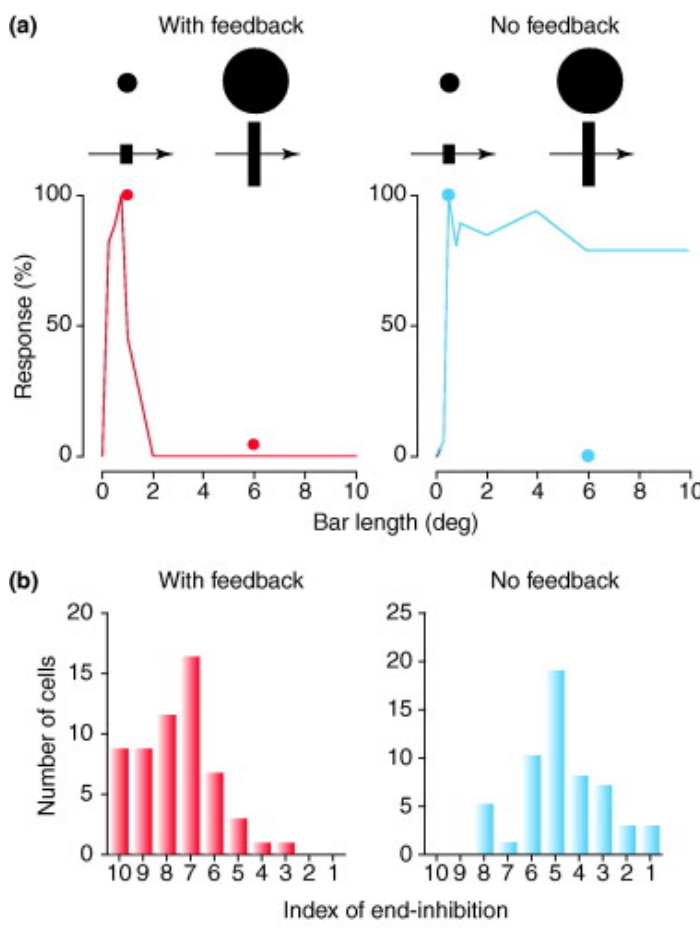

TRENDS in Neuroscience

Figure 2. Influence of corticofugal feedback on area summation properties of geniculate cells. (a) Comparison of the influence of corticofugal feedback on LGN cell responses to moving bars of varying length and to small and large stationary flashing spots. The tuning curves plot the responses of two LGN cells to drifting bars of varying length, and the responses to stationary flashing spots of light are denoted by the small circles. Bar length and spot diameter are represented (in degrees) along the $y$-axis. Stimuli are illustrated above the records. In the presence of feedback (left), the cell shows a very pronounced length tuning (red curve), responding selectively to a short 'optimal'-length bar crossing its receptive field. In the absence of feedback (right), this length tuning is substantially reduced (blue curve). By contrast, tests using a small and a large stationary flashing stimulus showed no difference in responses of the LGN cells between the with-feedback and without-feedback conditions. (b) Histograms summarizing the distribution of end-inhibition with (left) and without (right) feedback. Cells are grouped into ten categories for degree of endinhibition. Category 10 contains cells showing a total suppression of response at longer bar lengths whereas those in category 1 would show no suppression. See Refs 23 and 26 for further details. Adapted from Ref. [23].

Aside from the influences on the spatial properties of LGN-cell receptive fields, it is clear that feedback influences the firing pattern of LGN cells 22, 36, 37, 38 and 39 and also how their discharge might synchronize when several cells are coactivated by a common stimulus 37 and 39 . The synchronization of their discharge might be an epiphenomenon of the influence on receptive-field interactions, but the influence on firing pattern includes the ability of the feedback to shift cells between burst and tonic firing mode. However, an interesting point made by McClurkin et al. [22] is that the influence of feedback on the LGN enhances the information about the stimulus contained in the firing pattern. It is worth noting that where spikes are closely grouped in bursts or grouped by the synchronization of several inputs, the effective gain and security of the input to the cortex will be enhanced 40, 41, 42, 43 and 44. The mechanisms underlying these effects, and particularly the influence on the spatial properties of the receptive fields, are complex. However, they will depend on the metabotropic and ionotropic channels engaged by the feedback synapses onto relay cells and onto intrinsic and perigeniculate inhibitory interneurons 45, 46, 47 and 48 (Cudeiro and Sillito, in this issue). The feedback-driven factors influencing, for example, the preference of LGN cells for shorter bars (length-tuning or end-stopping in the LGN, as already discussed here and in Ref. [23]) might at first sight be linked simply to the gain of inhibitory mechanisms underlying the surround, but as Cudeiro and Sillito (in this issue; Ref. [48]) show, the process is more subtle and complex than this. In this sense, feedback fine-tunes local circuitry in the LGN to optimize the extraction of salient features, and in relation to the shift between burst and tonic firing might alert and focus the circuitry on stimuli requiring attention [36]. 
(a)
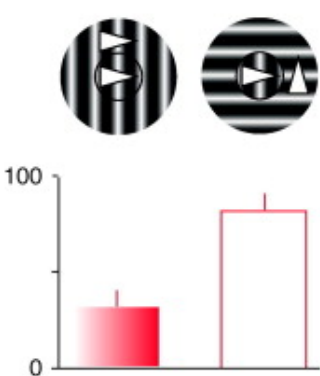

(b)

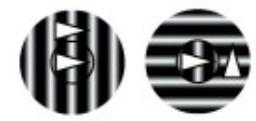

$\downarrow$

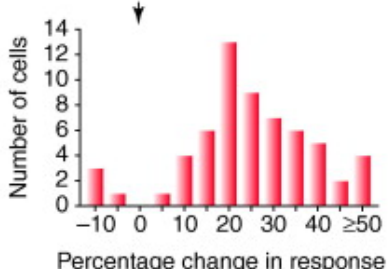

Percentage change in response
No feedback
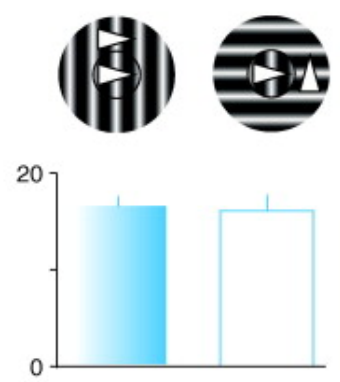

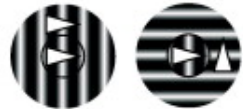

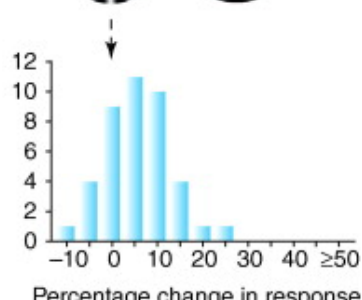

(c) With feedback

No feedback

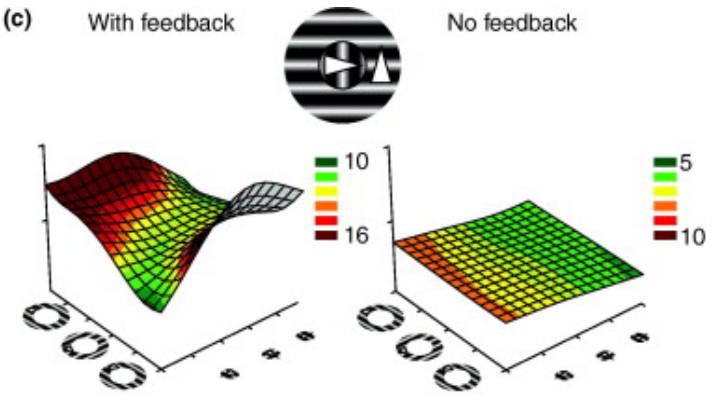

(d) Layer $4 \mathrm{C} \beta$ cell

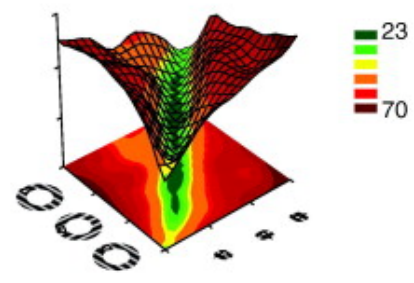

Figure 3. Corticofugal feedback enhances local pop-out effects in LGN cells. (a) Comparison of the response of an LGN cell to stimulation of the receptive-field centre using a patch of drifting grating in the presence of a surround grating either at the same orientation as the centre or orthogonal to it (as illustrated above the histogram bars). With feedback, the LGN cell discriminates between these conditions; without feedback, it does not. Histograms show response in spikes per second plus 1 SE. (b) Effects of feedback sampled across a group of cells with and without feedback. The histograms plot the percentage change in response observed between iso-oriented and orthogonally oriented surround configurations (normalized with respect to the centre-only response) in the presence (left) and absence (right) of corticofugal feedback. The mean increase in response magnitude for the switch from iso- to cross-oriented surround was $24.3 \%$ in the presence of feedback and this reduced to $5.6 \%$ without feedback. See Refs 24 and 25 for further details. (c) LGN-cell sensitivity to orientation contrast. The surface plot on the left shows the response of an LGN cell (in the presence of feedback) to an inner patch of grating of varying the orientation in the presence of an outer patch of grating also of varying orientation. The diagonal running from bottom left to top right represents all points where the orientation of the centre and surround stimuli were the same, over a complete sequence of absolute orientations. Response magnitude is shown by the height and shading of the contour. At all orientations, the responses were larger when orientation of the outer and inner stimulus differed. In the absence of feedback this effect is not seen (right). The colour scale bar represents response values in spikes per second. (d) Example of a non-oriented layer 4C $\beta$ cortical cell studied using the same paradigm as the LGN cells in (c). Note the similarity in the pattern to that of the LGN cell in the presence of feedback. Is this processing step deferred to layer 4C in the primate, and does it depend on the collaterals from layer 6 cells to layer 4? See Ref. [32] for further details. Colour scale bar represents response in spikes per second. Adapted from Refs 24, 25 and 32.

It is important to emphasize the visual specificity of the feedback to the LGN. Local drug-induced changes of visual response magnitude in layer 6 of V1 provoke shifts in firing pattern and receptive-field properties in LGN cells 26, 27, 38, 49 and 50. Layer 6 feedback cells are selective for orientation and direction, and their axonal arborizations in the LGN extend asymmetrically in retinotopic space along the axis of their preferred orientation and a path orthogonal to that reflecting the direction of motion 19 and 26 (Figure 1b-c). All this suggests that the feedback might be fine-tuned to address local processing of motion and orientation or salient discontinuities defining fine-scale segmentation that are highlighted by motion and contour.

It is relevant here to consider briefly the effects of the axon collaterals of layer 6 cells on layer 4 cells in V1. One could argue that these are also in essence feedback connections [51]. In vitro, intracellular and in vivo extracellular studies indicate a facilitatory influence that tends to modulate rather than to drive the layer 4 cell response, at least at lower firing rates 52 and 53. This might under some circumstances bias the spatial properties of the layer 4 cell receptive fields 54, 55, 56 and 57. Assuming that the layer 6 cells contact cells in layer 4 that are in the same orientation column or in columns that have the same orientation preference, this would reinforce the stimulus characteristics that optimize the layer 4 cell responses. Together, these components of the effects of layer 6 cells underline the view that these cells exert a potent and stimulus-specific influence on the access of retinal input to the brain, by actions in LGN and cortical layer 4 . The access of feedback from MT to this circuitry suggests that its role in vision is broad, and highlights the fact that our understanding of it is crucial to any model of early visual mechanisms in perception. 


\section{Stimulus context and the influence of feedback from area MT}

While considering the significance of this influence, it is tempting to try and build an understanding based simply around local spatial properties of the layer 6 cell filter and the relationship of this filter to those of the cells contacted in the LGN and layer 4. However, it is now well documented that responses of V1 cells are strongly influenced by stimulus context 32, 33, 34, 58, 59 and 60. Consequently, for anything other than a simple discrete stimulus, the visual response characteristics of the receptive field that forms the spatiotemporal 'filter' driving the feedback will be significantly more complicated than a first-pass impression might suggest. This is brought into sharp focus by the projection from MT to V1 layer 6. MT receives fast input from the magnocellular system (largely via layer 4B of V1, but also via layer 6) and many of its cells are, among other things, direction selective 12 and 61. The receptive fields are much (up to ten times) larger than those of V1 cells 12 and 62 and for any given spatial location an MT receptive field centred over a V1 receptive field will subtend a significant area of visual space before and after the V1 field (Figure 4). The feedback projection from MT to V1 also connects to a retinotopic area of V1 that corresponds to the receptive-field size of the MT cell 14 and 63. Thus, a moving stimulus entering an MT receptive field, and causing it to respond, will start to drive a feedback influence that affects V1 cells at retinotopic locations ahead of and around the actual stimulus location. It might be argued that this primes V1 cells to behave differently when the stimulus reaches them or causes them to respond differently to another stimulus that is already driving their input. We suggest that V1 circuitry is modulated at retinal locations representing salient features of the larger-scale integration carried out in MT, to optimize processing of the pattern and direction of motion of the stimulus as a whole.

(a)<smiles>C[13CH]Br</smiles>

MT

Bar
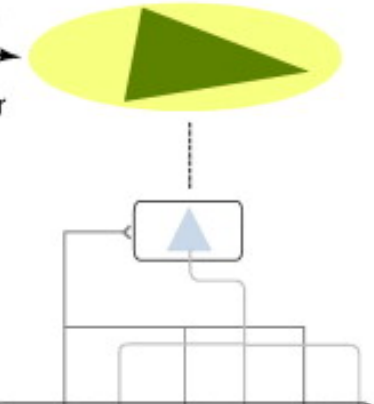

V1

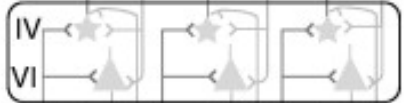

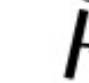

LGN

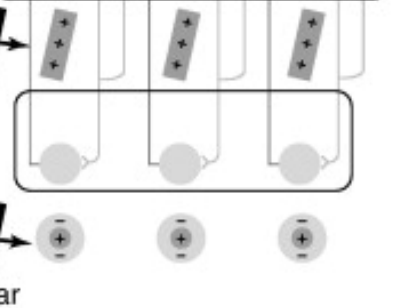

MT

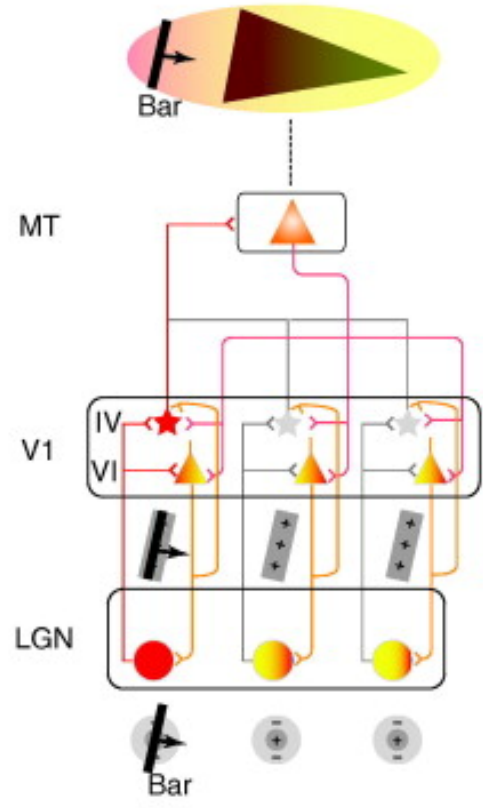

(b)

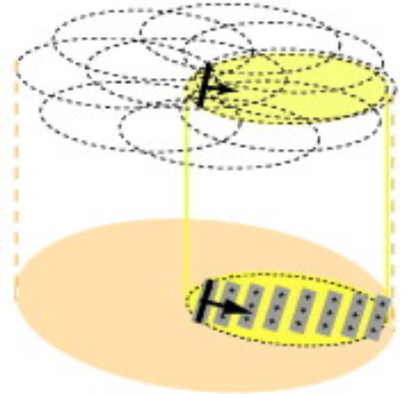

$\overline{\text { TRENDS in Neurosciences }}$

Figure 4. Feedback from MT has the capacity to influence V1 and LGN cells at retinotopic locations ahead of the current stimulus location. (a) How a moving stimulus will engage a wave of feedback ahead of its location, via connections up to MT and back down again via layer 6 of V1 to the LGN. Large fields at the top of the panel are those of an MT cell with its directional preference indicated by the large shaded arrowhead. The V1-cell and LGN-cell receptive fields are not to scale. Stimulus icons (bars) at all three levels reflect the same stimulus as it engages the receptive fields for the same spatial location in the local receptive fields. Shades of colour from red (strongly activated) through yellow to grey (no activation) indicate level of activation of the cells. (b) Partially shifted overlap of a group of MT cell receptive fields and how the whole group would be partially engaged by a single stimulus. Assuming that each MT cell projects to a retinotopic area of V1 that matches its receptive field, the solid yellow and dashed orange lines show respectively the area of V1 engaged by a single MT cell and the group activated by a single stimulus. See text for further discussion.

This raises the further question of whether, by changing the behaviour of cells in V1, MT feedback will influence the LGN and the consequent change in the LGN will form part of the process registering the pattern motion direction. In a timing sense, the logic of this is clear for locations ahead of the stimulus. However, it is pertinent that the speed of transmission through the magnocellular pathway to MT is such that straight up and down feedback from MT can influence retinal information that is being 
relayed through more slowly conducting parvocellular- and konicellular-dominated pathways as these inputs arrive at the LGN and V1 12, 64, 65 and 66. Consequently, the integration in MT might also modulate LGN circuitry to optimize the representation of stimulus pattern, as we have already discussed.

\section{Effects of MT on responses in V1}

Is there experimental evidence that MT can influence the visual responses of V1 cells? MT provides strong feedback to layer 4B of V1 in addition to layer 6 so there are strong grounds for believing that it can. And indeed it does. Hupe et al. compared the response of V1 cells to moving and flashed visual stimuli when feedback from MT was either present or inactivated by focal cooling [66], and they found a significant difference in V1-cell responses with and without the feedback. It is particularly interesting that the influence of MT was apparent in the first $10 \mathrm{~ms}$ of the response to the stimulus driving the V1 cell, even for flashed stimuli. One could argue that this approach led to a false conclusion about the influence of MT feedback on V1 because cooling simply lifted a background influence that led to a global statedependent change in V1 circuitry, with an impact on all visual responses. However a different approach, involving a highly localized iontophoretic application of a $\mathrm{GABA}_{\mathrm{B}}$ receptor antagonist to MT cells ( Figure 5), has also revealed strong effects on V1-cell responses, supporting the work of Hupe et al. [66]. In this study 27, 67 and 68, the $\mathrm{GABA}_{\mathrm{B}}$ antagonist was applied at a dose that did not change spontaneous activity but did increase visual response magnitude. The experiment essentially compared responses of multiple single cells simultaneously recorded in V1 under control conditions and when there was a local focal increase in visual response magnitude in MT, as monitored by recording from a cell at the site of the focal drug application. This revealed the influence of MT in terms of the change provoked by a focal increase in MT response magnitude (without change in specificity of MT-cell responses). The effects observed were reversible and repeatable. The experiments revealed differential effects on V1 cells - some showed response increases and others response decreases - and these effects linked to receptive-field characteristics for simple and non-oriented cells. Furthermore, some cells showed significant changes in how stimulus context influenced responses to stimuli overlying their receptive field. This suggested a significant shift in how the system was handling these interactions and indicated, at least for moving stimuli, that the normal characteristics of these interactions 27, 32, 33, 34, 58, 59, 60, 66, 67 and 68 draw on the influence of MT. In this sense, the characteristics of V1-cell responses that might be integrated in the larger MT-cell receptive fields and contribute to their direction selectivity 13, 35 and 69 might also in part depend on feedback. Hence, the focus of the mechanism contributing to the perception of motion direction derives from the interaction between levels.

\section{Effects of MT in the LGN}

Because MT influences V1-cell responses and V1 influences LGN-cell responses, are changes in LGN processing driven by changes in visual responses in MT? This was examined in experiments similar to those of Jones et al. 27, 67 and 68, but in which recordings were made of multiple single cells in the LGN 26 and 70 ( Figure 5). The results were clear: a small focal change in visual response magnitude in MT produced significant changes in the response magnitude of LGN cells. These experiments did not examine visual response properties of the LGN cells in detail, but did demonstrate that even a small change in response magnitude in MT produced effects on LGN-cell response magnitude and firing pattern. These effects were seen in magnocellular, parvocellular and konicellular LGN cells. Hence, feedback from MT influences early sensory processing in both V1 and LGN. Because of the size of MTcell receptive fields, for stimuli crossing the field the state of the circuitry is altered in a substantive retinotopic area of V1 and in the LGN underlying the MT field. Allowing for a partially shifted overlap of MT-cell receptive fields (which means a stimulus will coactivate many MT cells simultaneously, reflecting a larger area of retinotopic feedback to V1; Figure 4), a stimulus in motion will influence the neural networks and hence visual processing in even larger retinotopic areas of V1 and the LGN (with effects on parvocellular, magnocellular and konicellular channels) than would be expected if a single MTcell receptive field were considered ( Figure 4). 
(a)
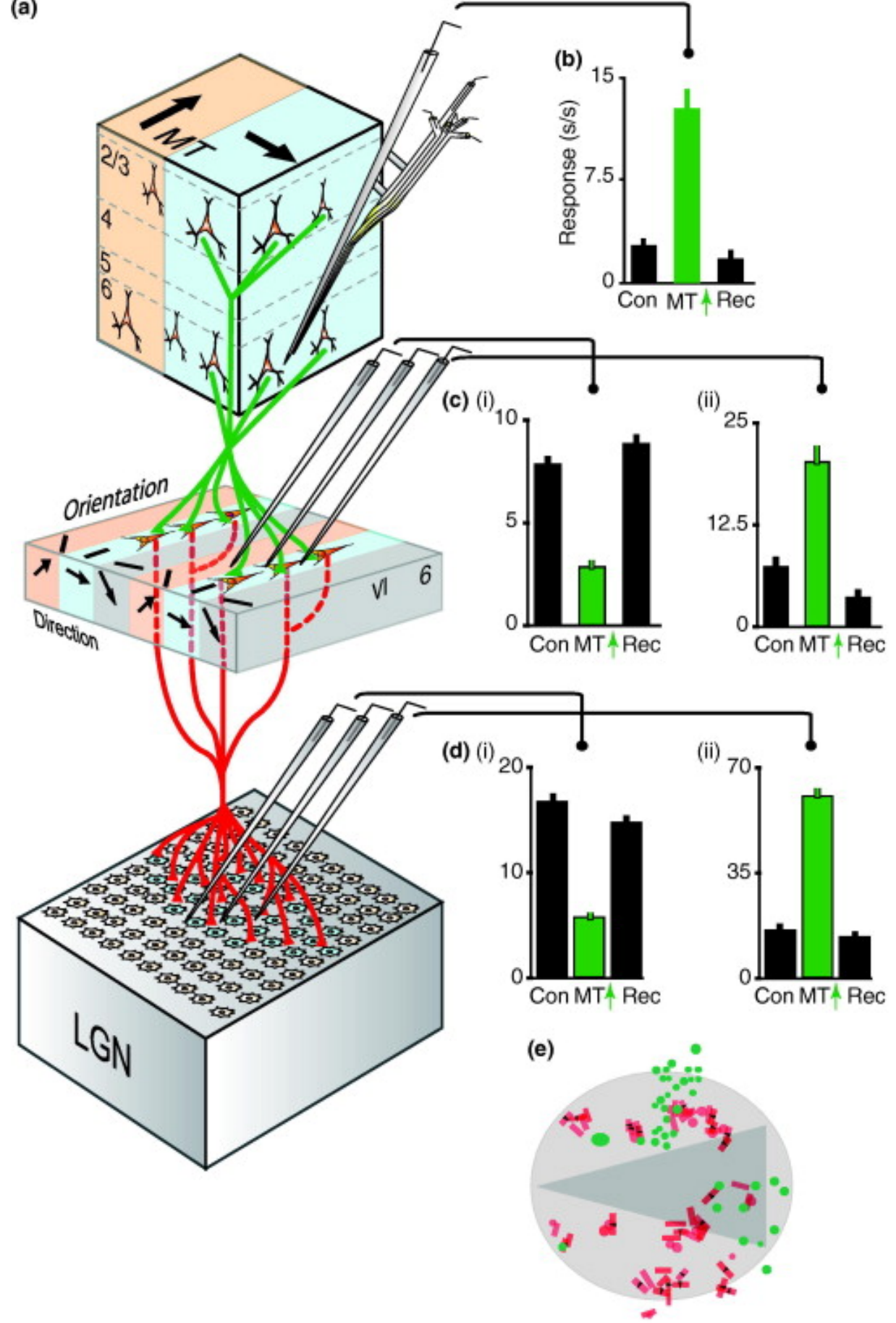

TRENDS in Neurosciences

Figure 5. MT feedback influences V1-cell and LGN-cell responses to visual stimulation. (a) Composite diagram showing experimental design. A combined iontophoretic pipette for drug delivery and recording electrode is inserted into MT, and arrays of single electrodes to make simultaneous recordings from three or more single cells are inserted into V1 and the LGN. A small iontophoretic current of the $\mathrm{GABA}_{\mathrm{B}}$ receptor antagonist CGP enhances visual response magnitude (b) but not background firing in the MT cell. This results in changes in response magnitude in V1 cells (c) and LGN cells (d) to the same stimulus that is driving the MT cell. Enhancement of MT feedback could evoke either increased (c,ii and d,ii) or decreased (c,i and d,i) responses in both V1 and the LGN. For LGN cells, there did not appear to be any correlation between the type of effect observed (i.e. increase or decrease) and cell type (parvocellular, magnocellular or koniocellular). For V1, most simple cells showed an increase in response magnitude whereas the responses of the majority of non-oriented cells decreased during enhancement of MT feedback. (e) An averaged MT field, with the LGN (green) and V1 (red) cells studied superimposed to scale. 


\section{Feedback from MT and perception}

MT cells are sensitive to direction of stimulus motion, to the speed of a stimulus and to the depth of a stimulus in visual space 12 and 13. Many also possess suppressive surrounds which, among other things, might underlie sensitivity to surface slope and object motion in direction and depth 12, 13, 71 and 72. However, these properties are not all generated de novo in MT; for example, sensitivity to the stimulus direction and depth can be directly linked back to the properties of V1 cells 13 and 69. The MT cell in this sense can be argued to simply integrate information from V1 cells over a wider area of visual space. To take the example of the direction of object motion, because the receptive fields of direction-selective V1 cells are small their response can be ambiguous with respect to the real direction of motion of a larger stimulus: they only 'see' a component of the contours of the object translating over their 'window on the world' (the aperture problem [13]). However strongly end-stopped cells tend to be suppressed by such stimuli (and thus provide a diminished output to higher levels), except when their receptive field or 'window on the world' lies at the edge of such contours, they then provide (because they are tracking salient contour or pattern ends) an unambiguous response with respect to object direction. Recent work 13 and 35 suggests that the output of these end-stopped cells is integrated by MT cells to generate their selectivity. Similar arguments apply to other properties, notably sensitivity to object depth.

So MT draws on V1 responses, but responses in V1 are strongly influenced by feedback from MT. Hence MT can be argued to shape the response of the cells providing its input. In this sense, one might consider that the selectivity at both levels result from the iterative interaction between them. This might already be seen as a step too far by many, but we suggest a further step to the LGN. To recapitulate, the feedback down the neuraxis from MT is fast. It originates from directional cells and is, as we discussed earlier, directed to the LGN via layer 6 cells that are themselves directionally selective and have asymmetric axonal arborizations in the LGN that link to the spatial characteristics of their receptive fields. Additionally, the feedback effects from V1 seem to be most clearly seen in the LGN for moving stimuli 23 and 73, not stationary stimuli. All this argues that a feedback influence reaching the LGN particularly focuses on objects in motion and on the direction of motion. Given our comments earlier in this review on end-stopping and the aperture problem, it is particularly interesting that feedback from V1 to the LGN enhances that property in LGN cells. Does this contribute to end-stopping in V1, or does it constitute a separate layer of finer spatial focus, simply highlighting the input from LGN cells that represent the edges of the pattern?

The selectivity of cells in MT and V1 to pattern motion evolves over time 35 and 74 and seems linked to the development of surround suppression and/or end-stopping in V1-cell receptive fields. We suggest this reflects the time course of iterative interactions among MT, V1 and the LGN. Feedback from MT will engage the circuitry in V1 and the LGN in a way that reflects the 'characteristics' of the stimulus driving MT, and by this token restructure the earlier processing mechanism to optimize representation of the predicted trajectory, and indeed its predicted associations in visual space behind and to the side of the stimulus. As we have already suggested, the output from the LGN might be highlighted by cells with receptive fields that lie along the termination points of the edges of a pattern in motion, and feedback might strengthen this and focus the circuitry on the overall shape of the moving object that is extracted by MT cells. The key point is that information processing in the LGN changes as the activity of MT cells changes and this binds the LGN into the circuit that provides the perceptual basis for the moment-bymoment delineation of the object in motion.

Other work suggests that there is an iterative interaction between MT and V1 in motion perception. Several studies 75, 76 and 77 using transcranial magnetic stimulation (TMS) in human subjects underline the role that feedback from MT has on processing in V1 and perception. Recently, Silvanto et al. 76 and 77 examined the visual phosphenes (sensations of light in response to non-visual stimuli) produced by TMS applied to MT and V1 separately or together, with various time intervals between applications in the dual-stimulation paradigm. The results are best explained by the suggestion that feedback from MT influences how activity in V1 is perceived and indeed further suggest that the information content in V1 determines whether that information reaches consciousness. However interpreted, these data give a clear indication that feedback from MT to V1 influences the perceptual interpretation of the visual input engaging V1. One might exclude the LGN from this process but attentional changes have been seen in both macaque and human LGN 78 and 79 and have been linked in a model to the feedback from layer 6 [80]. In what is possibly an even closer link to perception, Haynes et al. have reported eye-specific effects of binocular rivalry in the human LGN [81]. Thus, many strands of evidence indicate that dynamic changes in LGN function are linked to perception. 


\section{Final comment}

The visual world is neither stationary nor involves single stimuli presented out of context. It is a world in motion and a complex world, with many components, some linked and some independent, that are evolving, shifting and changing. The eyes move, the body moves and the visual world moves. The nature of the circuitry, synaptic actions and effects we review here suggest that this visual world is reflected in an integrated, essentially simultaneous modulation and coordination of filters in the circuitry. These filters hold the representation at each of what might have been regarded as successive processing steps in the system, except that they are not separate steps; they are one system locking onto the visual world in motion. However, a key point is that the patterns of activation depend on how specific image components engage the system and these produce focal but interacting shifts, both across and between levels. Overall, one would suggest that a particular feature of the feedback running from MT and then V1 to the LGN might be to adjust local circuitry, to highlight the output of cells at points representing the salient edges of objects and to suppress the output from cells that do not carry this information (this might link to the way in which MT cells handle noise [13]). This will lead to a sparse output encoding the stimulus and, we suggest, enable multiple representations of different objects moving across each other and in depth. Why is feedback necessary for this? Simply, because it is only at higher levels such as MT that larger-scale integration is carried out, and feedback from these regions can inform the earlier levels about the broad borders of an object in ways that are inaccessible to the circuits at these levels. How can it be fine tuned? By the object borders engaging the local receptive fields after a broad-brush modulation of the cells lying along the outline, as defined by the feedback. This is a process that categorizes and integrates the different components of the visual world in motion and enables both fine-scale resolution and large-scale integration in a way that underpins the needs of processes such as transparent motion and the extraction of salience from camouflage.

\section{References}

1 Erisir, A. et al . (1997) Relative numbers of cortical and brainstem inputs to the lateral geniculate nucleus. Proc. Natl. Acad. Sci. U. S. A. 94, 1517-1520

2 Van Horn, S.C. et al . (2000) Relative distribution of synapses in the A-laminae of the lateral geniculate nucleus of the cat. J. Comp. Neurol. 416, 509-520

3 Wilson, J.R. (1993) Circuitry of the dorsal lateral geniculate nucleus in the cat and monkey. Acta Anat. (Basel) 147, 1-13

4 Sherman, S.M. and Guillery, R.W. (2002) The role of the thalamus in the flow of information to the cortex. Philos. Trans. R. Soc. Lond. B Biol. Sci. 357, 1695-1708

5 Boyapati, J. and Henry, G.H. (1984) Corticofugal axons in the lateral geniculate nucleus of the cat. Exp. Brain Res. 53, 335-340

6 Murphy, P.C. and Sillito, A.M. (1996) Functional morphology of the feedback pathway from area 17 of the cat visual cortex to the lateral geniculate nucleus. J. Neurosci. 16, 1180-1192

7 Murphy, P.C. et al . (2000) Comparison of the laminar distribution of input from areas 17 and 18 of the visual cortex to the lateral geniculate nucleus of the cat. J. Neurosci. 20, 845-853

8 Robson, J.A. (1983) The morphology of corticofugal axons to the dorsal lateral geniculate nucleus in the cat. J. Comp. Neurol. 216, 89-103

9 Ahmed, B. et al . (1994) Polyneuronal innervation of spiny stellate neurons in cat visual cortex. J. Comp. Neurol. 341, 39-49

10 Latawiec, D. et al . (2000) Termination of the geniculocortical projection in the striate cortex of macaque monkey: a quantitative immunoelectron microscopic study. J. Comp. Neurol. 419, 306-319

11 Orban, G.A. (1994) Motion processing in monkey striate cortex. In Cerebral Cortex (Vol. 10) (Peters, A. and Rockland, K.S., eds), pp. 413-441, Plenum Press

12 Orban, G.A. (1997) Visual processing in macaque area MT/V5 and its satellites (MSTd and MSTv). In Cerebral Cortex (Vol. 12) (Rockland, K.S. et al. , eds), pp. 359-379, Plenum Press

13 Born, R.T. and Bradley, D.C. (2005) Structure and function of visual area MT. Annu. Rev. Neurosci. 28, 157-189

14 Shipp, S. and Zeki, S. (1989) The organization of connections between areas V5 and V1 in macaque monkey visual cortex. Eur. J. Neurosci. 1, 309-332

15 Rockland, K.S. and Knutson, T. (2000) Feedback connections from area MTof the squirrel monkey to areas V1 and V2. J. Comp. Neurol. 425, 345-368

16 Schiller, P.H. et al . (1990) Functions of the colour-opponent and broad-band channels of the visual system. Nature 343, 68-70 
17 Casagrande, V.A. (1994) A third parallel visual pathway to primate area V1. Trends Neurosci. 17, 305-309

18 Grieve, K.L. and Sillito, A.M. (1995) Differential properties of cells in the feline primary visual cortex providing the corticofugal feedback to the lateral geniculate nucleus and visual claustrum. J. Neurosci. 15, 4868-4874

19 Murphy, P.C. et al. (1999) Feedback connections to the lateral geniculate nucleus and cortical response properties. Science 286, 1552-1554

20 Tsumoto, T. et al. (1978) Functional organization of the corticofugal system from visual cortex to lateral geniculate nucleus in the cat. Exp. Brain Res. 32, 345-364

21 Marrocco, R.T. et al. (1982) Modulation of lateral geniculate nucleus cell responsiveness by visual activation of the corticogeniculate pathway. J. Neurosci. 2, 256-263

22 McClurkin, J.W. et al. (1994) Cortical feedback increases visual information transmitted by monkey parvocellular lateral geniculate nucleus neurons. Vis. Neurosci. 11, 601-617

23 Murphy, P.C. and Sillito, A.M. (1987) Corticofugal feedback influences the generation of length tuning in the visual pathway. Nature 329, 727-729

24 Sillito, A.M. et al. (1993) Orientation sensitive elements in the corticofugal influence on centresurround interactions in the dorsal lateral geniculate nucleus. Exp. Brain Res. 93, 6-16

25 Cudeiro, J. and Sillito, A.M. (1996) Spatial frequency tuning of orientation-discontinuity-sensitive corticofugal feedback to the cat lateral geniculate nucleus. J. Physiol. 490, 481-492

26 Sillito, A.M. and Jones, H.E. (2002) Corticothalamic interactions in the transfer of visual information. Philos. Trans. R. Soc. Lond. B Biol. Sci. 357, 1739-1752

27 Sillito, A.M. and Jones, H.E. (2003) Feedback systems in visual processing. In The Visual Neurosciences (Chalupa, L.M. and Werner, J.S., eds), pp. 609-624, MIT Press

28 Jones, H.E. and Sillito, A.M. (1991) The length-response properties of cells in the feline dorsal lateral geniculate nucleus. J. Physiol. 444, 329-348

29 Jones, H.E. et al. (2000) Spatial summation in lateral geniculate nucleus and visual cortex. Exp. Brain Res. 135, 279-284

30 Hayot, F. and Tranchina, D. (2001) Modeling corticofugal feedback and the sensitivity of lateral geniculate neurons to orientation discontinuity. Vis. Neurosci. 18, 865-877

31 Webb, B.S. et al. (2002) Feedback from V1 and inhibition from beyond the classical receptive field modulates the responses of neurons in the primate lateral geniculate nucleus. Vis. Neurosci. 19, 583592

32 Jones, H.E. et al. (2002) Spatial organization and magnitude of orientation contrast interactions in primate V1. J. Neurophysiol. 88, 2796-2808

33 Sillito, A.M. et al. (1995) Visual cortical mechanisms detecting focal orientation discontinuities. Nature 378, 492-496

34 Jones, H.E. et al. (2001) Surround suppression in primate V1. J. Neurophysiol. 86, 2011-2028

35 Pack, C.C. et al. (2003) End-stopping and the aperture problem: two-dimensional motion signals in macaque V1. Neuron 39, 671-680

36 Sherman, S.M. (2001) Tonic and burst firing: dual modes of thalamocortical relay. Trends Neurosci. 24, 122-126

37 Sillito, A.M. et al. (1994) Feature-linked synchronization of thalamic relay cell firing induced by feedback from the visual cortex. Nature 369, 479-482

38 Worgotter, F. et al. (1998) The influence of corticofugal feedback on the temporal structure of visual responses of cat thalamic relay cells. J. Physiol. 509, 797-815

39 Kirkland, K.L. et al. (2000) Oscillations and long-lasting correlations in a model of the lateral geniculate nucleus and visual cortex. J. Neurophysiol. 84, 1863-1868

40 Larkum, M.E. et al. (1999) A new cellular mechanism for coupling inputs arriving at different cortical layers. Nature 398, 338-341

41 Roy, S.A. and Alloway, K.D. (2001) Coincidence detection or temporal integration? What the neurons in somatosensory cortex are doing. J. Neurosci. 21, 2462-2473

42 Miller K, D. (1994) A model for the development of simple cell receptive fields and the ordered arrangement of orientation columns through activity-dependent competition between ON and OFFcenter inputs. J. Neurosci. 14, 409-441

43 Pinto, D.J. et al. (2003) Cortical damping: Analysis of thalamocortical response transformations in rodent barrel cortex. Cereb. Cortex 13, 33-44

44 Usrey, W.M. (2002) The role of spike timing for thalamocortical processing. Curr. Opin. Neurobiol. 12, 411-417 
45 Godwin, D.W. et al. (1996) Ultrastructural localization suggests that retinal and cortical inputs access different metabotropic glutamate receptors in the lateral geniculate nucleus. J. Neurosci. 16, 81818192

46 Salt, T.E. (2002) Glutamate receptor functions in sensory relay in the thalamus. Philos. Trans. R. Soc. Lond. B Biol. Sci. 357, 1759-1766

47 Von Krosigk, M. et al. (1999) Dynamic properties of corticothalamic excitatory postsynaptic potentials and thalamic reticular inhibitory postsynaptic potentials in thalamocortical neurons of the guinea-pig dorsal lateral geniculate nucleus. Neuroscience 91, 7-20

48 Cudeiro, J. and Sillito, A.M. (2006) Looking back: corticothalamic feedback and early visual processing. Trends Neurosci. doi: 10.1016/j.tins.2006.05.002

49 Wang, W. et al.(2000) Influence of enhanced feedback from V1 on visual responses in the LGN. Program number 162.9. In 2000 Abstract Viewer and Itinerary Planner , Society for Neuroscience Online (http://sfn.scholarone.com/)

50 Wang, W.et al.(2004) Functional alignment of feedback effects from visual cortex to LGN. Program number 409.11. In 2004 Abstract Viewer and Itinerary Planner , Society for Neuroscience Online (http://sfn.scholarone.com/)

51 Callaway, E.M. (1998) Local circuits in primary visual cortex of the macaque monkey. Annu. Rev. Neurosci. 21, 47-74

52 Ferster, D. and Lindstrom, S. (1985) Augmenting responses evoked in area 17 of the cat by intracortical axon collaterals of cortico-geniculate cells. J. Physiol. 367, 217-232

53 Tarczy-Hornoch, K. et al. (1999) Intracortical excitation of spiny neurons in layer 4 of cat striate cortex in vitro. Cereb. Cortex 9, 833-843

54 Bolz, J. and Gilbert, C.D. (1986) Generation of end-inhibition in the visual cortex via interlaminar connections. Nature 320, 362-365

55 Grieve, K.L. and Sillito, A.M. (1991) The length summation properties of layer VI cells in the visual cortex and hypercomplex cell end zone inhibition. Exp. Brain Res. 84, 319-325

56 Grieve, K.L. and Sillito, A.M. (1991) A re-appraisal of the role of layer VI of the visual cortex in the generation of cortical end inhibition. Exp. Brain Res. 87, 521-529

57 Grieve, K.L. and Sillito, A.M. (1995) Non-length-tuned cells in layers II/III and IV of the visual cortex: The effect of blockade of layer VI on responses to stimuli of different lengths. Exp. Brain Res. $104,12-20$

58 Knierim, J.J. and Van Essen, D.C. (1992) Neuronal responses to static texture patterns in area V1 of the alert macaque monkey. J. Neurophysiol. 67, 961-980

59 Gilbert, C.D. and Wiesel, T.N. (1990) The influence of contextual stimuli on the orientation selectivity of cells in primary visual cortex of the cat. Vision Res. 30, 1689-1701

60 Lamme, V.A. (1995) The neurophysiology offigure-ground segregation in primary visual cortex. J. Neurosci. 15, 1605-1615

61 Maunsell, J.H. and Newsome, W.T. (1987) Visual processing in monkey extrastriate cortex. Annu. Rev. Neurosci. 10, 363-401

62 Albright, T.D. and Desimone, R. (1987) Local precision of visuotopic organization in the middle temporal area (MT) of the macaque. Exp. Brain Res. 65, 582-592

63 Salin, P.A. and Bullier, J. (1995) Corticocortical connections in the visual system: structure and function. Physiol. Rev. 75, 107-154

64 Raiguel, S. et al. (1989) Response latencies of visual cells in macaque areas V1 V2 and V5. Brain Res. 493, 155-159

65 Schmolesky, M.T. et al. (1998) Signal timing across the macaque visual system. J. Neurophysiol. 79, 3272-3278

66 Hupe, J.M. et al. (2001) Feedback connections act on the early part of the responses in monkey visual cortex. J. Neurophysiol. 85, 134-145

67 Jones, H.E. et al.(2000) Influence of enhanced MT feedback on the responses of cells in primate V1. Program number 162.10. In 2000 Abstract Viewer and Itinerary Planner, Society for Neuroscience Online (http://sfn.scholarone.com/)

68 Jones, H.E. et al.(2001) Spatial characteristics and laminar organization of feedback effects from MT to V1. Program number 620.11. In 2001 Abstract Viewer and Itinerary Planner, Society for Neuroscience Online (http://sfn.scholarone.com/)

69 Pack, C.C. et al. (2006) Spatiotemporal structure of nonlinear subunits in macaque visual cortex. J. Neurosci. 26, 893-907

70 Jones, H.E. et al.(2002) MT feedback effects in the primate LGN. Program number 658.17. In 2002 Abstract Viewer and Itinerary Planner, Society for Neuroscience Online (http://sfn.scholarone.com/) 
71 Raiguel, S. et al. (1995) Shape and spatial distribution of receptive fields and antagonistic motion surrounds in the middle temporal area (V5) of the macaque. Eur. J. Neurosci. 7, 2064-2082

72 Xiao, D.K. et al. (1997) Selectivity of macaque MT/V5 neurons for surface orientation in depth specified by motion. Eur. J. Neurosci. 9, 956-964

73 Gulyás, B. et al. (1990) Corticofugal feedback influences the responses of geniculate neurons to moving stimuli. Exp. Brain Res. 79, 441-446

74 Pack, C.C. and Born, R.T. (2001) Temporal dynamics of a neural solution to the aperture problem in visual area MT of macaque brain. Nature 409, 1040-1042

75 Pascual-Leone, A. and Walsh, V. (2001) Fast backprojections from the motion to the primary visual area necessary for visual awareness. Science 292, 510-512

76 Silvanto, J. et al. (2005) Striate cortex (V1) activity gates awareness of motion. Nat. Neurosci. 8, 143144

77 Silvanto, J. et al. (2005) Double dissociation of V1 and V5/MT activity in visual awareness. Cereb. Cortex 15, 1736-1741

78 Vanduffel, W. et al. (2000) Attention-dependent suppression of metabolic activity in the early stages of the macaque visual system. Cereb. Cortex 10, 109-126

79 O’Connor, D.H. et al. (2002) Attention modulates responses in the human lateral geniculate nucleus. Nat. Neurosci. 5, 1203-1209

80 Orban, G.A. et al. (2005) Attentional suppression early in the macaque visual system. In Neurobiology of Attention (Itti, L. et al., eds), pp. 429-434, Academic Press, Elsevier

81 Haynes, J.D. et al. (2005) Eye-specific effects of binocular rivalry in the human lateral geniculate nucleus. Nature 438, 496-499 